\title{
Time-resolved anisotropic coherent anti-Stokes Raman scattering: A new probe of reorientational dynamics
}

\author{
Chaozhi Wan and Carey K. Johnson \\ Department of Chemistry, University of Kansas, Lawrence, Kansas 66045
}

(Received 21 June 1993; accepted 10 August 1993)

\begin{abstract}
A formalism for the time-dependent anisotropic third-order susceptibility induced by a linearly polarized excitation pulse has been derived to describe the time dependence of coherent anti-Stokes Raman scattered (CARS) and Raman time-resolved experiments. The third-order susceptibility induced in a randomly oriented molecular system contains nine independent molecular parameters-three isotropic and six anisotropic - as compared to three independent parameters in an isotropic system. Methods of time-resolved anisotropic anti-Stokes Raman scattering (TRA CARS) are discussed as an approach to reorientational measurements. The dependence of the TRA CARS or TRA Raman signal on vibration and polarization component can in principle provide more rotational information than fluorescence depolarization or linear dichroism measurements and may allow more complete characterization of rotational dynamics. Results are reported for three types of TRA CARS experiments to demonstrate the capabilities of TRA CARS to study the rotational motion of molecules in liquids.
\end{abstract}

\section{INTRODUCTION}

When an isotropic molecular system is excited by linearly polarized light, anisotropic distributions of the ground and excited states are induced. This anisotropy decays due to rotational motion and population decay of the molecules. The time-dependent anisotropy can be measured by time-resolved spectroscopies, including fluorescence depolarization, time-resolved linear dichroism, polarization spectroscopy, and transient grating spectroscopy. ${ }^{1-7}$

The time-dependent anisotropy can also be probed by higher-order spectroscopies. Since the excitation (pump) pulse in pump-probe time-resolved coherent and spontaneous Raman techniques is typically linearly polarized, the time-delayed probe pulse or pulses interrogate an anisotropic orientational distribution. Consequently, symmetry rules for elements of the Raman scattering tensor $\alpha_{i j}$ or the susceptibility $\chi_{i j k l}^{(3)}$ in isotropic systems are no longer valid. For example, in isotropic media, $\chi_{1111}^{(3)}=\chi_{2222}^{(3)}$ and $\alpha_{11}$ $=\alpha_{22}$. These relations are in general invalid in systems excited by a polarized pump pulse. As a result, timeresolved Raman and time-resolved coherent anti-Stokes Raman (CARS) signals may exhibit a time dependence due to rotation or anisotropy decay, and this time dependence can be exploited to acquire vibrationally specific information on reorientational motions. In this paper, we treat specifically the case of rotational relaxation by rotational diffusion. However, the experimental techniques described can be applied more broadly to the rotational relaxation of chromophores, whether or not the dynamics can be described by small-step rotational diffusion models.

Anisotropy decay by rotational diffusion contains in the most general case five exponentials for an asymmetric rotor, ${ }^{8}$ which reduce to three and one exponentials for symmetric and spherical rotors, respectively. However, in practice, only single exponential decays are observed for many molecules, most of which are not symmetric rotors. ${ }^{9}$
Clearly, the conventional methods of detecting rotational diffusion cannot resolve multiple exponential decays when the decay time constants are similar. In order to specify further the components of the diffusion tensor, additional experimental information is required. Spontaneous Raman linewidths and depolarizational light-scattering measurements in the frequency domain have provided another probe of reorientational correlation functions. ${ }^{10,11}$ However, the molecular rotational relaxation parameters extracted from these measurements depend on the deconvolution of other contributions to the bandwidth, which can lead to serious complications, particularly for asymmetric vibrations. ${ }^{11}$ Furthermore, frequency-domain Raman experiments probe only the rotational invariants of an isotropic system. In contrast, the CARS methods discussed here probe anisotropic as well as isotropic components of the third-order nonlinear optical susceptibility.

The CARS signal, is described by the third-order susceptibility $\chi_{i j k l}\left(-\omega_{3} ; \omega_{0}, \omega_{1},-\omega_{2}\right)$, where the labels $(i, j$, $k$, and $l$ ) describe the dependence of the CARS signal on the polarization of the incident and detected light. In fluorescence depolarization and linear dichroism, one detects the anisotropy of a transition dipole. However, higherorder anisotropy is also induced by excitation with polarized light, e.g., anisotropy of the third-order susceptibility. This anisotropy can be detected in time-resolved coherent or spontaneous Raman spectroscopy. Consequently, timeresolved Raman spectroscopy can in principle provide further information to determine more completely the rotational motion of molecules in liquids. The time dependence of the anisotropic component of the nonlinear susceptibility will in fact contribute to time-resolved Raman or CARS signals along with the vibrational dynamics which such experiments may be intended to probe. The theory of time-resolved resonance Raman anisotropy has been the subject of a recent communication. ${ }^{12}$ In this paper, we derive expressions for the time dependence of the anisotropic CARS and Raman susceptibility and demonstrate the ap- 
plication of TRA CARS to the rotational motion of dye molecules. Our goal is to characterize time-resolved anisotropic anti-Stokes Raman scattering (TRA CARS) and spontaneous Raman (TRA Raman) as probes of rotational motion.

\section{THEORY}

When a sample system is illuminated by three laser beams at frequencies $\omega_{0}, \omega_{1}$, and $\omega_{2}$, the incident fields induce a third-order polarization of the medium

$$
P^{(3)}\left(\omega_{3}\right)=\chi^{(3)}\left(-\omega_{3}, \omega_{0}, \omega_{1},-\omega_{2}\right) E\left(\omega_{0}\right) E\left(\omega_{1}\right) E^{*}\left(\omega_{2}\right)
$$

at the CARS frequency $\omega_{3}=\omega_{0}+\omega_{1}-\omega_{2} . \quad E\left(\omega_{i}\right)$ denotes the amplitude of the laser field at frequency $\omega_{i}$, and $\chi^{(3)}$ is the third-order optical susceptibility tensor of the medium. In a Cartesian coordinate system, the $i$ th component $P_{i}^{(3)}$ of $\mathbf{P}^{(3)}$ is related to the components $E_{j}, E_{k}$, and $E_{l}$ of the laser amplitudes by ${ }^{13,14}$

$$
\begin{aligned}
P_{i}^{(3)}\left(\omega_{3}\right)= & \frac{6}{m !} \sum_{j k l} \chi_{i j k l}\left(-\omega_{3}, \omega_{0}, \omega_{1},-\omega_{2}\right) \\
& \times E_{j}\left(\omega_{0}\right) E_{k}\left(\omega_{1}\right) E_{l}^{*}\left(\omega_{2}\right),
\end{aligned}
$$

where $m$ is the number of identical frequencies; $m=2$ for frequency-degenerate CARS $\left(\omega_{0}=\omega_{1}\right)$. The third-order polarization is a source of coherent radiation at $\omega_{3}$. The CARS intensity radiated by the $i$ th component of the third-order polarization is

$$
\begin{gathered}
I_{i}\left(\omega_{3}\right)=C \mid \sum_{j k l} \chi_{i j k l}\left(-\omega_{3}, \omega_{0}, \omega_{1},-\omega_{2}\right) \\
\times\left. E_{j}\left(\omega_{0}\right) E\left(\omega_{1}\right) E^{*}\left(\omega_{2}\right)\right|^{2},
\end{gathered}
$$

where $C$ is a constant depending on laser frequencies and spatial geometry. The third-order susceptibility $\chi_{i j k l}$ may be written

$$
\chi_{i j k l}\left(-\omega_{3}, \omega_{1}, \omega_{1},-\omega_{2}\right)=C^{\prime} \frac{\beta_{i j}^{*} \alpha_{k l}}{\omega_{b a}-\omega_{1}+\omega_{2}+i \gamma_{b a}}
$$

for frequency-degenerate CARS with $\omega_{0}=\omega_{1}$; where $C^{\prime}$ is a constant, $\omega_{b a}=\left(E_{b}-E_{a}\right) / \hbar$, where $E_{b}$ and $E_{a}$ are the energies of states $b$ and $a$, and $\gamma_{b a}$ is the rate of dephasing between levels $b$ and $a$. The polarizability tensor elements for anti-Stokes and Stokes Raman scattering $\beta_{i j}^{*}$ and $\alpha_{k l}$ are given by

$\beta_{i j}^{*}=\sum_{n^{\prime}}\left(\frac{\left\langle a\left|\mu_{i}\right| n^{\prime}\right\rangle\left\langle n^{\prime}\left|\mu_{j}\right| b\right\rangle}{\omega_{n^{\prime} b}-\omega_{1}-i \gamma_{n^{\prime} b}}+\frac{\left\langle a\left|\mu_{j}\right| n^{\prime}\right\rangle\left\langle n^{\prime}\left|\mu_{i}\right| b\right\rangle}{\omega_{n^{\prime} b}+\omega_{3}+i \gamma_{n^{\prime} b}}\right)$,

$\alpha_{k l}=\sum_{n}\left(\frac{\left\langle b\left|\mu_{l}\right| n\right\rangle\left\langle n\left|\mu_{k}\right| a\right\rangle}{\omega_{n a}-\omega_{1}-i \gamma_{n a}}+\frac{\left\langle b\left|\mu_{k}\right| n\right\rangle\left\langle n\left|\mu_{l}\right| a\right\rangle}{\omega_{n a}+\omega_{2}-i \gamma_{n a}}\right)$,

where $a$ is the initial level, $b$ is the vibrational level, and $n$ and $n^{\prime}$ are the intermediate levels.

\section{A. The induced anisotropic susceptibility}

In time-resolved anisotropic CARS a linearly polarized laser pulse at frequency $\omega_{e}$ passes through the isotropic sample, creating anisotropically oriented excited-state populations and leaving an orientational "hole" in the ground state. Various combinations of polarizations of the CARS input pulses propagating parallel to the pump beam can be selected to detect the CARS polarization components of interest. The third-order susceptibility $\chi_{i j k l}\left(-\omega_{3} ; \omega_{0}, \omega_{1}\right.$, $-\omega_{2}$ ) is a fourth rank tensor with 81 components. In isotropic media, the macroscopic third-order susceptibility tensor has 21 nonzero components, of which only three are independent. After excitation by a linearly polarized laser pulse, the medium becomes anisotropic and the number of independent components of the susceptibility tensor increases due to the induced anisotropic third-order susceptibility.

To derive the time-dependent anisotropic third-order optical susceptibility, we need first to define a moleculefixed axis system, which for convenience is taken to coincide with the principal axes of the diffusion tensor. The orientation of the molecule-fixed axes with respect to the laboratory axes is specified by the three Euler angles $(\alpha, \beta, \gamma) \equiv \Omega$. For an $n$-level molecular system, a normalized probability distribution $f^{(i)}(\Omega, t)$ describes the orientational distribution for each level, ${ }^{3}$ where $f^{(i)}(\Omega, t) d \Omega$ is the probability of finding a molecule in level $i$ with orientation between $\Omega$ and $\Omega+d \Omega$ at time $t$ and

$$
N^{(i)}(t)=\frac{1}{8 \pi^{2}} \int d \Omega f^{(i)}(\Omega, t)
$$

is the number density of molecules in level $i$.

We define the susceptibility of a molecule in level $i$ with orientation $\Omega$ as $M_{i j k l}^{(i)}(\Omega)$ in the laboratory-fixed axis system. Then the time-dependent third-order nonlinear susceptibility of the molecular system in level $i$ can be written

$$
\chi_{i j k l}^{(i)}(t)=\frac{1}{8 \pi^{2}} \int d \Omega M_{i j k l}^{(i)}(\Omega) f^{(i)}(\Omega, t) .
$$

The distribution function $f^{(i)}(\Omega, t)$ has been discussed by a number of authors. ${ }^{1-4}$ Its evolution for an $n$-level system undergoing rotational diffusion is determined by the diffusion equation and the level kinetics ${ }^{3}$

$$
\begin{aligned}
\frac{\partial}{\partial t} f^{(i)}(\Omega, t)= & -\sum_{\alpha=1}^{3} R_{\alpha}^{(i)} L_{\alpha}^{2} f^{(i)}(\Omega, t) \\
& +\sum_{j=0}^{n-1} k_{i j} f^{(j)}(\Omega, t) .
\end{aligned}
$$

Here $R_{\alpha}^{(i)}$ are the diffusion tensor elements in the principal axis systems $(\alpha=1,2,3)$ for level $i, L_{\alpha}$ are the angularmomentum operators with respect to the molecule-fixed axis system, and $k_{i j}$ are the set of first-order rate constants describing transitions between the levels. Two terms contribute to the change in probability density in level $i$ at $\Omega$ - one resulting from diffusional motion of molecules in level $i$ and a second resulting from molecules with orien- 
tation $\Omega$ making transitions to and from level $i$. This set of coupled equations has been solved in terms of the asymmetric rotor wave functions $\Psi_{l \tau m}^{(i)}$, which are eigenfunctions of the diffusion operator $\Sigma_{\alpha}\left(R_{\alpha}^{(i)} L_{\alpha}^{2}\right)$. In the case of electric dipole induced transitions, $f^{(i)}(\Omega, t)$ can be expanded in a basis of six asymmetric rotor wave functions. Then the asymmetric rotor wave functions can in turn be expanded in terms of the symmetric rotor wave functions. With these expansions, the orientational distribution function for level $i$ generated by the pump pulse becomes ${ }^{1,3}$

$$
\begin{aligned}
f^{(i)}(\Omega, t)= & a_{00}^{(i)}(t)+\left(\frac{8 \pi^{2}}{5}\right)^{1 / 2} \sum_{\tau=-2}^{2} a_{2 \tau}^{(i)}(t) \\
& \times \sum_{T=-2}^{2} A_{2 \tau T}^{(i)} \Phi_{T 0}^{(2)}(\Omega),
\end{aligned}
$$

where the expansion coefficients $A_{2 \tau T}^{(i)}$ are tabulated elsewhere ${ }^{15,16}$ and $\Phi_{K M}^{(L)}(\Omega)$ are the symmetric rotor eigenfunctions related to the Wigner functions $D_{M K}^{(L)}(\Omega)$ by ${ }^{17}$

$$
\Phi_{K M}^{(L)}(\Omega)=\left(\frac{2 L+1}{8 \pi^{2}}\right)^{1 / 2} D_{M K}^{(L) *}(\Omega)
$$

The coefficients $a_{00}^{(i)}(t)$ describe the time evolution of the isotropic component of the molecular system and contain only the level kinetics, while the five coefficients $a_{2 \tau}^{(t)}(t)$ describe the time dependence of the anisotropic components and are responsible for the rotational decay. For a two-level system where the ground and excited states have the same rotational diffusion constants, we have ${ }^{3}$

$$
\begin{aligned}
& a_{00}^{(0)}(t)=N_{0}-N^{(1)}(0) \exp \left(-k_{f} t\right), \\
& a_{00}^{(1)}(t)=N^{(1)}(0) \exp \left(-k_{f} t\right), \\
& a_{2 \tau}^{(0)}(t)=a_{2 \tau}^{(0)}(0) \exp \left[-\left(k_{f}+E_{2 \tau}^{(0)}\right) t\right], \\
& a_{2 \tau}^{(1)}(t)=a_{2 \tau}^{(1)}(0) \exp \left[-\left(k_{f}+E_{2 \tau}^{(1)}\right) t\right],
\end{aligned}
$$

where

$$
\begin{aligned}
& a_{2 \tau}^{(1)}(0)=-a_{2 \tau}^{(0)}(0), \\
& a_{2 \tau}^{(1)}(0)=N^{(1)}(0) \sqrt{6} \sum_{T=-2}^{2} F_{T}^{(2)} A_{2 \tau T}^{(1)}
\end{aligned}
$$

with

$$
\begin{aligned}
& F_{0}^{(2)}=\frac{1}{\sqrt{6}}\left(3 \mu_{z}-1\right), \quad F_{ \pm 1}^{(2)}=\mp\left(\mu_{x} \pm i \mu_{y}\right) \mu_{z}, \\
& F_{ \pm 2}^{(2)}=\frac{1}{2}\left(\mu_{x} \pm i \mu_{y}\right)^{2} .
\end{aligned}
$$

In Eqs. (12)-(17), $N_{0}$ is the total number density and $N^{(1)}(0)$ is the initial number density of the excited state prepared by the excitation pulse; $k_{f}$ is the kinetic decay rate of the excited state; $E_{2 \tau}^{(2)}$ are the eigenvalues of the rotational diffusion operator; ${ }^{2,16}$ and $\mu_{x}, \mu_{y}$, and $\mu_{z}$ are the projections of the unit transition dipole along the moleculefixed axes.

Substituting Eqs. (10) and (11) into Eq. (8), we have

$$
\chi_{i j k l}^{(i)}(t)=a_{00}^{(i)}(t) \chi_{i j k l, 00}^{(i)}+\sum_{\tau=-2}^{2} a_{2 \tau}^{(i)}(t) \chi_{i j k l, 2 \tau}^{(i)},
$$

where

$$
\begin{aligned}
& \chi_{i j k l, 00}^{(i)}=\frac{1}{8 \pi^{2}} \int M_{i j k l}^{(i)}(\Omega) d \Omega \\
& \chi_{i j k l, 2 \tau}^{(i)}=\sum_{T=-2}^{2} A_{2 \tau T}^{(i)} \frac{1}{8 \pi^{2}} \int M_{i j k l}^{(i)}(\Omega) D_{0 T}^{(2) *}(\Omega) d \Omega .
\end{aligned}
$$

The first and second terms in Eq. (18) describe the isotropic and anisotropic decays of the third-order nonlinear response, respectively.

In the next section, we calculate the orientation averages in Eqs. (19) and (20) to obtain the anisotropic nonlinear susceptibility $\chi_{i j k l}^{(i)}(t)$.

\section{B. The orientational average}

Irreducible spherical tensor formalism permits ready calculation of the orientational average of the third-order susceptibility tensor in Eqs. (19) and (20). In general, a fourth rank tensor $\chi_{i j k l}$ can be decomposed into irreducible tensors as

$$
\Gamma\left(\chi_{i j k l}\right)=3 \Gamma^{(0)} \oplus 6 \Gamma^{(1)} \oplus 6 \Gamma^{(2)} \oplus 3 \Gamma^{(3)} \oplus \Gamma^{(4)} .
$$

Since the integrals in Eqs. (19) and (20) are nonzero only when $M_{i j k l}^{(i)}(\Omega)$ contains components belonging to the $\Gamma^{(0)}$ or $\Gamma^{(2)}$ irreducible representation, one can immediately conclude that TRA CARS is described by a nonlinear susceptibility tensor containing three independent components in the $L=0$ subspace and six independent components in the $L=2$ subspace.

First we write the molecular susceptibility $M_{i j k l}^{(i)}(\Omega)$ in terms of the direct product of two second rank tensors $\beta_{i j}^{(i) *}(\Omega)$ and $\alpha_{k l}^{(i)}(\Omega)$,

$$
M_{i j k l}(\Omega)=\beta_{i j}^{*}(\Omega) \alpha_{k l}(\Omega),
$$

where we have dropped the label for the level $i$ for simplicity. The polarizability tensor elements $\beta_{i j}^{*}(\Omega)$ and $\alpha_{k l}(\Omega)$ describe anti-Stokes and Stokes Raman scattering, respectively. The second rank tensors $\beta_{i j}^{*}(\Omega)$ and $\alpha_{k l}(\Omega)$ in Cartesian coordinates can be written in terms of the spherical tensor components $\beta_{P}^{(L)^{*}}$ and $\alpha_{P^{\prime}}^{\left(L^{\prime}\right)}$ by a unitary transformation $^{18} U=U(i j, L P)$,

$$
\begin{gathered}
\beta_{i j}^{*}(\Omega)=\sum_{L=0}^{2} \sum_{P=-L}^{L} U^{*}(i j, L P) \beta_{P}^{(L) *}(\Omega), \\
\alpha_{k l}(\Omega)=\sum_{L^{\prime}=0}^{2} \sum_{P^{\prime}=-L^{\prime}}^{L^{\prime}} U\left(k l, L^{\prime}, P^{\prime}\right) \alpha_{P^{\prime}}^{\left(L^{\prime}\right)}(\Omega),
\end{gathered}
$$

or by the reverse transformation $U^{-1}(L P, i j)$ $=U^{*}(i j, L P)$,

$$
\begin{aligned}
& \beta_{P}^{(L)^{*}(\Omega)}=\sum_{i, j=X, Y, Z} U(i j, L P) \beta_{i j}^{*}(\Omega), \\
& \alpha_{P^{\prime}}^{\left(L^{\prime}\right)}(\Omega)=\sum_{k, l=X, Y, Z} U^{*}\left(k l, L^{\prime}, P^{\prime}\right) \alpha_{k l}(\Omega) .
\end{aligned}
$$


TABLE I. The elements $U(i j, L P)$ of the unitary transformation from Cartesion to spherical tensor components.

\begin{tabular}{|c|c|c|c|c|c|c|c|c|c|}
\hline \multirow[b]{2}{*}{$i j$} & \multicolumn{9}{|c|}{$L P$} \\
\hline & 00 & 11 & 10 & $1-1$ & 22 & 21 & 20 & $2-1$ & $2-2$ \\
\hline$x x$ & $-1 / 3$ & & & & $1 / 2$ & & $-1 / \sqrt{ } 6$ & & $1 / 2$ \\
\hline$y y$ & $-1 / 3$ & & & & $-1 / 2$ & & $-1 / \sqrt{ } 6$ & & $-1 / 2$ \\
\hline$z z$ & $-1 / 3$ & & & & & & $2 / \sqrt{6}$ & & \\
\hline$x y$ & & & $-i / 2$ & & $-i / 2$ & & & & $i / 2$ \\
\hline$y x$ & & & $i / 2$ & & $-i / 2$ & & & & $i / 2$ \\
\hline$x z$ & & $-1 / 2$ & & $-1 / 2$ & & $-1 / 2$ & & $1 / 2$ & \\
\hline$z x$ & & $1 / 2$ & & $1 / 2$ & & $-1 / 2$ & & $1 / 2$ & \\
\hline$y z$ & & $i / 2$ & & $-i / 2$ & & $i / 2$ & & $i / 2$ & \\
\hline$z y$ & & $-i / 2$ & & $i / 2$ & & $i / 2$ & & $i / 2$ & \\
\hline
\end{tabular}

Table I shows the transformation elements $U(i j, L P)$, which have also appeared elsewhere. ${ }^{18,19}$

The spherical tensor components $\beta_{P}^{(L) *}(\Omega)$ and $\alpha_{p e}^{\left(L^{\prime}\right)}(\Omega)$ in laboratory-fixed axes are transformed to molecule-fixed axes by the rotational transformation ${ }^{20}$

$$
\begin{aligned}
& \beta_{P}^{(L) *}(\Omega)=\sum_{M=-L}^{L} D_{P M}^{(L)}(\Omega) \beta_{M}^{(L) *}, \\
& \alpha_{P^{\prime}}^{\left(L^{\prime}\right)}(\Omega)=\sum_{M^{\prime}=-L^{\prime}}^{L^{\prime}} D_{P^{\prime} M^{\prime}}^{\left(L^{\prime}\right) *}(\Omega) \alpha_{M^{\prime}}^{\left(L^{\prime}\right)},
\end{aligned}
$$

where the orientation angle $\Omega$ appears only in the Wigner functions $D_{P M}^{(L)}(\Omega)$. The direct product $\beta_{M}^{(L)^{*}} \alpha_{M^{\prime}}^{\left(L^{\prime}\right)}$ can be further reduced in terms of irreducible spherical tensors, but the transformation is unnecessary since the orientational averages in Eqs. (19) and (20) can be calculated from the orthogonality relations that characterize the Wigner functions ${ }^{21}$

$$
\begin{aligned}
& \int D_{P M}^{(L)}(\Omega) D_{P^{\prime} M^{\prime}}^{\left(L^{\prime}\right) *}(\Omega) d \Omega=\frac{8 \pi^{2}}{2 L+1} \delta_{L L^{\prime}} \delta_{P P^{\prime}} \delta_{M M^{\prime}}, \\
& \int D_{P M^{\prime}}^{(L)}(\Omega) D_{P^{\prime} M^{\prime}}^{\left(L^{\prime}\right) *}(\Omega) D_{0, T}^{(2) *} d \Omega \\
& =\frac{8 \pi^{2}}{5}(-1)^{M^{\prime}-P^{\prime}} C\left(L^{\prime} L 2 ;-P^{\prime} P 0\right) \\
& \quad \times C\left(L^{\prime} L 2 ;-M^{\prime} M T\right),
\end{aligned}
$$

where $C\left(L^{\prime} L 2 ;-P^{\prime} P 0\right)$ and $C\left(L^{\prime} L 2 ;-M^{\prime} M T\right)$ are the Clebsch-Gordan coefficients.

Substituting Eqs. (22)-(24) into Eqs (19) and (20), and simplifying with Eqs. (29) and (30), we obtain the isotropic susceptibility

$$
\chi_{i j k l, 00}=\sum_{L=0}^{2} S_{i j k l, L} G_{L},
$$

where

$$
S_{i j k l, L}=\frac{1}{2 L+1} \sum_{P=-L}^{L} U^{*}(i j, L P) U(k l, L P),
$$

$$
G_{L}=\sum_{M=-L}^{L} \beta_{M}^{(L) *} \alpha_{M}^{(L)}
$$

and the anisotropic susceptibility

$$
\chi_{i j k l, 2 \tau}=\sum_{L=0}^{2} \sum_{L^{\prime}=0}^{2} \frac{1}{5} S_{i j k l, L L^{\prime}} G_{L L^{\prime}}(\tau),
$$

where

$$
\begin{aligned}
S_{i j k l, L L^{\prime}}= & \sum_{P=-L}^{L} \sum_{P^{\prime}=-L^{\prime}}^{L^{\prime}}(-1)^{-P^{\prime}} C\left(L^{\prime} L 2 ;-P^{\prime} P 0\right) \\
& \times U^{*}(i j, L P) U\left(k l, L^{\prime} P^{\prime}\right) \\
G_{L L^{\prime}}(\tau)= & \sum_{T=-2}^{2} A_{\tau, T}^{(2)} \sum_{M=-L}^{L} \sum_{M^{\prime}=-L^{\prime}}^{L^{\prime}}(-1)^{M^{\prime}} \\
& \times C\left(L^{\prime} L 2 ;-M^{\prime} M T\right) \beta_{M}^{(L) *} \alpha_{M^{\prime}}^{\left(L^{\prime}\right)}
\end{aligned}
$$

The nonzero coefficients $S_{i j k l, L}$ and $S_{i j k l, L L^{\prime}}$ in the isotropic and anisotropic third-order susceptibilities $\chi_{i j k l, 00}$ and $\chi_{i j k l, 2 \tau}$ are listed in Table II. The three components $G_{L}$ are the rotational invariants, while the anisotropic constants $G_{L L^{\prime}}(\tau)$ are responsible for the rotational decay. Table III shows the expressions for $G_{L L^{\prime}}(\tau)$. If the polarizability tensors for anti-Stokes and Stokes Raman scatterings $\beta_{i j}^{*}$ and $\alpha_{k l}$ are known, the anisotropic constants $G_{L L^{\prime}}(\tau)$ can be obtained directly from these expressions.

\section{Time-resolved anisotropic CARS}

Two sources contribute to time dependence of anisotropic CARS susceptibilities in Eq. (18)-population kinetics and rotational dynamics, both contained in the anisotropic parameters $a_{2 \tau}^{(i)}(t)$ [Eqs. (14)-(17)]. Although ideally one would like to measure purely the rotational dynamics or population kinetics, anisotropic CARS signals contain both contributions.

Both the isotropic and anisotropic components of the CARS tensor [Eqs. (31) and (34)] consist of 21 nonzero components. Eight of these components describe configurations where the excitation pulse and the CARS probe pulses propagate along the same axis. From Table II, we can see that the differences $\chi_{1111}-\chi_{2222}, \chi_{1221}-\chi_{2112}, \chi_{1122}$ $-\chi_{2211}$, and $\chi_{1212}-\chi_{2121}$ contain only the anisotropic part of the CARS tensor while the sums $\chi_{1111}+2 \chi_{2222}, \chi_{1221}$ $+\chi_{2112}+\chi_{2332}, \quad \chi_{1122}+\chi_{2211}+\chi_{2233}, \quad$ and $\chi_{1212}+\chi_{2121}$ $+\chi_{2323}$ contain only the isotropic part of the CARS tensor. Analogous with fluorescence depolarization, an anisotropy can be defined

$$
r(t)=\frac{\chi_{1111}(t)-\chi_{2222}(t)}{\chi_{1111}(t)+2 \chi_{2222}(t)}
$$

Similar anisotropies can be defined from other differences and sums. These anisotropies contain only rotational decays in the case of an isolated CARS transition. Unfortunately, measurement of these anisotropies is complicated by the fact that the CARS signal is proportional to the square of the susceptibility. Optical heterodyne techniques might provide a means to measure signals linear in the 
TABLE II. Coefficients in isotropic and anisotropic CARS tensor components $\chi_{i j k l, 00}$ and $\chi_{i j k l, 2 \tau}$.

\begin{tabular}{|c|c|c|c|c|c|c|c|c|c|c|c|c|c|c|}
\hline \multirow[b]{2}{*}{$\chi_{i j k l}$} & \multirow[b]{2}{*}{$E_{p}\left(\omega_{\mathrm{ex}}\right)$} & \multirow[b]{2}{*}{$E_{j}\left(\omega_{1}\right)$} & \multirow[b]{2}{*}{$E_{k}\left(\omega_{1}\right)$} & \multirow[b]{2}{*}{$E_{l}\left(\omega_{2}\right)$} & \multirow[b]{2}{*}{$E_{i}\left(\omega_{\mathrm{as}}\right)$} & \multicolumn{3}{|c|}{$\frac{S_{i j k l, L}}{L}$} & \multicolumn{6}{|c|}{$\begin{array}{c}S_{i j k l_{1}, L L^{\prime}} \\
L L^{\prime}\end{array}$} \\
\hline & & & & & & 0 & $\underline{1}$ & 2 & 02 & 20 & 11 & 12 & 21 & 22 \\
\hline$\chi_{1111}$ & $\uparrow$ & $\uparrow$ & $\uparrow$ & $\uparrow$ & $\uparrow$ & $\frac{1}{3}$ & $\cdots$ & $\frac{2}{15}$ & $-\frac{\sqrt{2}}{3}$ & $-\frac{\sqrt{2}}{3}$ & $\cdots$ & $\cdots$ & $\cdots$ & $-\frac{4}{3 \sqrt{14}}$ \\
\hline$\chi_{2222}$ & $\rightarrow$ & $\uparrow$ & $t$ & $\uparrow$ & $\uparrow$ & $\frac{1}{3}$ & $\cdots$ & $\frac{2}{15}$ & $\frac{\sqrt{2}}{6}$ & $\frac{\sqrt{2}}{6}$ & $\cdots$ & $\because$ & $\cdots$ & $\frac{2}{3 \sqrt{14}}$ \\
\hline$\chi_{1111}-\chi_{2222}$ & & & & & & $\cdots$ & $\cdots$ & $\cdots$ & $-\frac{1}{\sqrt{2}}$ & $-\frac{1}{\sqrt{2}}$ & $\therefore$ & $\cdots$ & $\cdots$ & $-\frac{2}{\sqrt{14}}$ \\
\hline$\chi_{1111}+2 \chi_{2222}$ & & & & & & 1 & $\cdots$ & $\frac{2}{5}$ & $\cdots$ & $\ldots$ & $\cdots$ & $\cdots$ & $\cdots$ & $\cdots$ \\
\hline$\chi_{1122}$ & $\uparrow$ & $\uparrow$ & $\rightarrow$ & $\rightarrow$ & $\uparrow$ & $\frac{1}{3}$ & $\cdots$ & $-\frac{1}{15}$ & $\frac{\sqrt{2}}{6}$ & $-\frac{\sqrt{2}}{3}$ & $\cdots$ & $\cdots$ & $\cdots$ & $\frac{2}{3 \sqrt{14}}$ \\
\hline$\chi_{2211}$ & $\rightarrow$ & $\uparrow$ & $\rightarrow$ & $\rightarrow$ & $\uparrow$ & $\frac{1}{3}$ & $\cdots$ & $-\frac{1}{15}$ & $-\frac{\sqrt{2}}{3}$ & $\frac{\sqrt{2}}{6}$ & $\cdots$ & $\cdots$ & $\cdots$ & $\frac{2}{3 \sqrt{14}}$ \\
\hline$\chi_{2233}$ & $\rightarrow$ & $\uparrow$ & $\odot$ & $\odot$ & $\uparrow$ & $\frac{1}{3}$ & $\ldots$ & $-\frac{1}{15}$ & $-\frac{\sqrt{2}}{6}$ & $\frac{\sqrt{2}}{6}$ & $\because$ & $\cdots$ & $\cdots$ & $-\frac{4}{3 \sqrt{14}}$ \\
\hline$\chi_{1122}-\chi_{2211}$ & & & & & & $\cdots$ & $\cdots$ & $\cdots$ & $\frac{1}{\sqrt{2}}$ & $-\frac{1}{\sqrt{2}}$ & $\ldots . . \cdots$ & $\cdots$ & $\cdots$ & $\cdots$ \\
\hline$\chi_{1122}+\chi_{2211}+\chi_{2233}$ & & & $\cdots$ & & & 1 & $\cdots$ & $-\frac{1}{5}$ & $\ldots$ & $\ldots$ & $\cdots$ & $\cdots$ & $\cdots$ & $\because$ \\
\hline$\chi_{1221}$ & $\uparrow$ & $\rightarrow$ & $\rightarrow$ & $\uparrow$ & 1 & $\cdots$ & $-\frac{1}{6}$ & $\frac{1}{10}$ & $\cdots$ & $\cdots$ & $\frac{1}{2 \sqrt{6}}$ & $-\frac{1}{2 \sqrt{2}}$ & $\frac{1}{2 \sqrt{2}}$ & $-\frac{1}{2 \sqrt{14}}$ \\
\hline$\chi_{2112}$ & $\rightarrow$ & $\rightarrow$ & $\rightarrow$ & $\uparrow$ & $-\uparrow$ & $\cdots$ & $-\frac{1}{6}$ & $\frac{1}{10}$ & $\cdots$ & $\cdots$ & $\frac{1}{2 \sqrt{6}}$ & $\frac{1}{2 \sqrt{2}}$ & $-\frac{1}{2 \sqrt{2}}$ & $-\frac{1}{2 \sqrt{14}}$ \\
\hline$\chi_{2332}$ & $\rightarrow$ & $\odot$ & $\odot$ & $\uparrow$ & $\uparrow$ & $\cdots$ & $=\frac{1}{6}$ & $\frac{1}{10}$ & $\cdots$ & $\cdots$ & $-\frac{1}{\sqrt{6}}$ & $\cdots$ & $\cdots$ & $\frac{1}{\sqrt{14}}$ \\
\hline$\chi_{1221}-\chi_{2112}$ & & & & & & $\cdots$ & $\cdots$ & $\cdots$ & $\cdots$ & $\cdots$ & $\cdots$ & $-\frac{1}{\sqrt{2}}$ & $\frac{1}{\sqrt{2}}$ & $\cdots$ \\
\hline$\chi_{1221}+\chi_{2112}+\chi_{2332}$ & & & & $\cdots$ & & $\cdots$ & $-\frac{1}{2}$ & $\frac{3}{10}$ & $\cdots$ & $\cdots$ & $\cdots$ & $\cdots$ & $\cdots$ & $\cdots$ \\
\hline$\chi_{1212}$ & $\uparrow$ & $\rightarrow$ & $\uparrow$ & $\rightarrow$ & $\uparrow$ & $\cdots$ & $\frac{1}{6}$ & $\frac{1}{10}$ & $\cdots$ & $\cdots$ & $-\frac{1}{2 \sqrt{6}}$ & $-\frac{1}{2 \sqrt{2}}$ & $-\frac{1}{2 \sqrt{2}}$ & $-\frac{1}{2 \sqrt{14}}$ \\
\hline$\chi_{2121}$ & $\rightarrow$ & $\rightarrow$ & $\uparrow$ & $\rightarrow$ & $\uparrow$ & $\cdots$ & $\frac{1}{6}$ & $\frac{1}{10}$ & $\cdots$ & $\cdots$ & $-\frac{1}{2 \sqrt{6}}$ & $\frac{1}{2 \sqrt{2}}$ & $\frac{1}{2 \sqrt{2}}$ & $-\frac{1}{2 \sqrt{14}}$ \\
\hline$\chi_{2323}$ & $\rightarrow$ & $\odot$ & $\uparrow$ & $\odot$ & $\uparrow$ & $\cdots$ & $\frac{1}{6}$ & $\frac{1}{10}$ & $\cdots$ & $\cdots$ & $\frac{1}{\sqrt{6}}$ & $\cdots$ & $\cdots$ & $\frac{1}{\sqrt{14}}$ \\
\hline$\chi_{1212}-\chi_{2121}$ & & & & & & $\cdots$ & $\cdots$ & $\cdots$ & $\cdots$ & $\cdots$ & $\cdots$ & $-\frac{1}{\sqrt{2}}$ & $-\frac{1}{\sqrt{2}}$ & $\cdots$ \\
\hline$\chi_{1212}+\chi_{2121}+\chi_{2323}$ & " & - & & & - & $\cdots$ & $\frac{1}{2}$ & $\frac{3}{10}$ & $\cdots$ & $\cdots$ & ${ }_{.} \ldots$ & $\cdots$ & $\cdots$ & $\cdots$ \\
\hline $\begin{array}{l}\chi_{1111}-\chi_{2222} \\
+\chi_{1122}-\chi_{2211} \\
+\chi_{1221}-\chi_{2112} \\
+\chi_{1212}-\chi_{2121}\end{array}$ & メ & $\uparrow$ & $\uparrow$ & $\uparrow$ & $\rightarrow$ & $\cdots$ & $\ldots$ & $\cdots$ & $\cdots$ & $-\sqrt{2}$ & $\cdots$ & $-\sqrt{2}$ & $\cdots$ & $-\frac{2}{\sqrt{14}}$ \\
\hline
\end{tabular}

susceptibility and thus determine the anisotropy. An example of optical heterodyne measurements is reported in Sec. III.

The time dependencies of the isotropic and anisotropic CARS susceptibilities are determined by the timedependent parameters $a_{2 \tau}(t)$, weighted in Eq. (18) by the isotropic and anisotropic susceptibility components $\chi_{i j k l, 00}$ and $\chi_{i j k l, 2 \tau}$, which are given by Eqs. (31) and (34). Hence, the relative contributions of each rotational decay component $E_{2 \tau}(t)$ are determined by the relative weights $\chi_{i j k l, 2 \tau}$ for each value of $\tau(\tau=-2, \ldots, 2)$ as determined by Eq. (34). These relative weights are dependent on the vibrational mode. Since the anisotropic components $G_{L L^{\prime}}(\tau)$ defined in Eq. (36) are vibration dependent, the time de- 
TABLE III. The anisotropic CARS components $G_{L L^{\prime}}(\tau){ }^{\mathrm{a}}$

$$
\begin{aligned}
& G_{02}(\tau)=A_{2 \tau} \beta_{0}^{(0) *} \alpha_{0}^{(2)}-A_{2 \tau 1} \beta_{0}^{(0) *} \alpha_{-1}^{(2)}-A_{2 \tau-1} \beta_{0}^{(0) *} \alpha_{1}^{(2)}+A_{2 \tau} \beta_{0}^{(0) *} \alpha_{-2}^{(2)}+A_{2 \tau-2} \beta_{0}^{(0) *} \alpha_{2}^{(2)} \\
& G_{20}(\tau)=A_{2 \tau} \beta_{0}^{(2) *} \alpha_{0}^{(0)}+A_{2 \tau 1} \beta_{1}^{(2) *} \alpha_{0}^{(0)}+A_{2 \tau-1} \beta_{-1}^{(2) *} \alpha_{0}^{(0)}+A_{2 \tau 2} \beta_{2}^{(2) *} \alpha_{0}^{(0)}+A_{2 \tau-2} \beta_{-2}^{(2)} * \alpha_{0}^{(0)}
\end{aligned}
$$

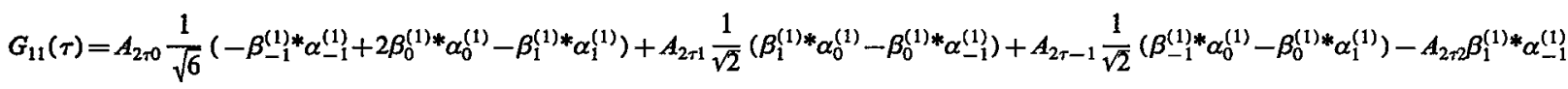

$$
\begin{aligned}
& -A_{2 \tau-2} \beta_{-1}^{(1) *} \alpha_{1}^{(1)} \\
& G_{12}(\tau)=A_{2 \tau 0} \frac{1}{\sqrt{2}}\left(\beta_{1}^{(1) *} \alpha_{1}^{(2)}-\beta_{-1}^{(1) *} \alpha_{-1}^{(2)}\right)+A_{2 \tau 1}\left(-\frac{1}{\sqrt{2}} \beta_{1}^{(1) *} \alpha_{0}^{(2)}-\frac{1}{\sqrt{6}} \beta_{0}^{(1) *} \alpha_{-1}^{(2)}+\frac{1}{\sqrt{3}} \beta_{-1}^{(1) *} \alpha_{-2}^{(2)}\right)+A_{2 \tau-1}\left(\frac{1}{\sqrt{2}} \beta_{-1}^{(1) *} \alpha_{0}^{(2)}+\frac{1}{\sqrt{6}} \beta_{0}^{(1) *} \alpha_{1}^{(2)}-\frac{1}{\sqrt{3}} \beta_{1}^{(1) *} \alpha_{2}^{(2)}\right) \\
& +A_{2+2}\left(\frac{\sqrt{2}}{\sqrt{3}} \beta_{0}^{(1) *} \alpha_{-2}^{(2)}+\frac{1}{\sqrt{3}} \beta_{1}^{(1) *} \alpha_{-1}^{(2)}\right)-A_{2 \tau-2}\left(\frac{\sqrt{2}}{\sqrt{3}} \beta_{0}^{(1) *} \alpha_{2}^{(2)}+\frac{1}{\sqrt{3}} \beta_{-1}^{(1) *} \alpha_{1}^{(2)}\right) \\
& G_{21}(\tau)=A_{2 * 0} \frac{1}{\sqrt{2}}\left(\beta_{1}^{(2) *} \alpha_{1}^{(1)}-\beta_{-1}^{(2)} * \alpha_{-1}^{(1)}\right)+A_{2 \tau 1}\left(-\frac{1}{\sqrt{6}} \beta_{1}^{(2) *} \alpha_{0}^{(1)}-\frac{1}{\sqrt{2}} \beta_{0}^{(2) *} \alpha_{-1}^{(1)}+\frac{1}{\sqrt{3}} \beta_{2}^{(2) *} \alpha_{1}^{(1)}\right)+A_{2 \tau-1}\left(\frac{1}{\sqrt{6}} \beta_{-1}^{(2) *} \alpha_{0}^{(1)}+\frac{1}{\sqrt{2}} \beta_{0}^{(2) *} \alpha_{1}^{(1)}-\frac{1}{\sqrt{3}} \beta_{-2}^{(2) *} \alpha_{-1}^{(1)}\right) \\
& -A_{2 \tau 2}\left(\frac{\sqrt{2}}{\sqrt{3}} \beta_{2}^{(2) *} \alpha_{0}^{(1)}+\frac{1}{\sqrt{3}} \beta_{1}^{(2) *} \alpha_{-1}^{(1)}\right)+A_{2 \tau-2}\left(\frac{\sqrt{2}}{\sqrt{3}} \beta_{-2}^{(2) *} \alpha_{0}^{(1)}+\frac{1}{\sqrt{3}} \beta_{-1}^{(2) *} \alpha_{1}^{(1)}\right)
\end{aligned}
$$

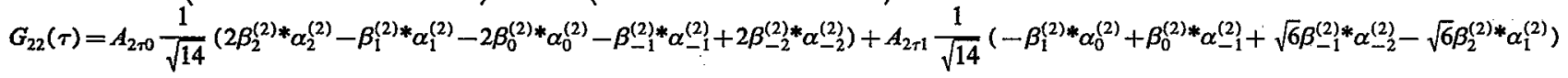

$$
\begin{aligned}
& +A_{2 \tau-1} \frac{1}{\sqrt{14}}\left(-\beta_{-1}^{(2) *} \alpha_{0}^{(2)}+\beta_{0}^{(2) *} \alpha_{1}^{(2)}+\sqrt{6} \beta_{1}^{(2) *} \alpha_{2}^{(2)}-\sqrt{6} \beta_{-2}^{(2) *} \alpha_{-1}^{(2)}\right)+A_{2 \tau 2}\left(\frac{\sqrt{2}}{\sqrt{7}} \beta_{2}^{(2) *} \alpha_{0}^{(2) *}+\frac{\sqrt{2}}{\sqrt{7}} \beta_{0}^{(2) *} \alpha_{-2}^{(2)}+\frac{\sqrt{3}}{\sqrt{7}} \beta_{1}^{(2) *} \alpha_{-1}^{(2)}\right) \\
& +A_{2 \tau-2}\left(\frac{\sqrt{2}}{\sqrt{7}} \beta_{0}^{(2) *} \alpha_{2}^{(2)}+\frac{\sqrt{2}}{\sqrt{7}} \beta_{-2}^{(2) *} \alpha_{0}^{(2)}+\frac{\sqrt{3}}{\sqrt{7}} \beta_{-1}^{(2) *} \alpha_{1}^{(2)}\right)
\end{aligned}
$$

${ }^{2}$ The coefficients $A_{2 \tau T}$ are given in Refs. 15 and 16.

pendence of TRA CARS signals also depends on the vibration. This vibrational selectivity in TRA CARS will in principle result in a vibration dependence in measured rotational decay times. In the following, we discuss the vibration dependence of the anisotropic constants $G_{L L^{\prime}}(\tau)$.

As an illustrative example, we consider two vibrational modes-for one (mode 1), the only nonzero components of the anti-Stokes and Stokes tensors are $\beta_{z z}^{*}$ and $\alpha_{z z}$; for mode 2 , the only nonzero components of the anti-Stokes and Stokes tensors are $\beta_{x z}^{*}$ and $\alpha_{z x}$, respectively. These types of anti-Stokes and Stokes tensors are possible in the case of resonance CARS for molecules with $C_{2}, C_{2 h}, C_{2 v}$, $D_{2}$, and $D_{2 h}$ symmetries. ${ }^{18}$ The nonzero CARS anisotropic constants for these vibrational modes are

$$
G_{02}(\tau)=G_{20}(\tau)=\frac{\sqrt{7}}{2} G_{22}(\tau)=-\frac{\sqrt{2}}{3} A_{\tau, 0}^{(2)} \beta_{z z}^{*} \alpha_{z z}
$$

for mode 1 and

$$
\begin{aligned}
G_{11}(\tau) & =-\sqrt{\frac{7}{3}} G_{22}(\tau) \\
& =\left(\frac{1}{2 \sqrt{6}} A_{\tau, 0}^{(2)}+\frac{1}{4} A_{\tau, 2}^{(2)}+\frac{1}{4} A_{\tau,-2}^{(2)}\right) \beta_{x z}^{*} \alpha_{z x}, \quad(39) \\
G_{21}(\tau) & =-G_{12}(\tau) \\
& =\left(-\frac{1}{2 \sqrt{2}} A_{\tau, 0}^{(2)}+\frac{1}{4 \sqrt{3}} A_{\tau, 2}^{(2)}+\frac{1}{4 \sqrt{3}} A_{\tau,-2}^{(2)}\right) \beta_{x z}^{*} \alpha_{z x}
\end{aligned}
$$

for mode 2 . In both cases, the TRA CARS signal contains only two rotational decays with $\tau=0$ and 2 since the expansion coefficients $A_{\tau 0}^{(2)}$ and $A_{\tau 2}^{(2)}+A_{\tau-2}^{(2)}$ are nonzero only when $\tau=0$ and $2 .^{15,16}$ For a symmetric rotor, the TRA CARS signal further reduces to a single rotational decay with $\tau=0$ in the case of the mode 1 since $A_{20}^{(2)}$ is zero for a symmetric rotor. ${ }^{3}$

For mode 2, it is possible in some circumstances to find an experimental configuration to measure only the rotational decay with $\tau=2$. We consider a configuration where the CARS signal field $E\left(\omega_{a s}\right)$, the excitation laser field $E_{p}\left(\omega_{\mathrm{ex}}\right)$, and the CARS probe field $E\left(\omega_{2}\right)$ are parallel, while the CARS pump field $E\left(\omega_{1}\right)$ is polarized at an angle $\alpha$ from them. In this polarization configuration, the effective third-order optical susceptibility is

$$
\chi_{\mathrm{eff}}=\chi_{1111} \cos ^{2} \alpha+\chi_{1221} \sin ^{2} \alpha .
$$

Using Table II and Eqs. (34), (39), and (40), we obtain the anisotropic part of the effective susceptibility

$$
\begin{aligned}
\chi_{\mathrm{eff}, 2 \tau}= & \frac{1}{5}\left\{\left[\frac{1}{21} A_{\tau 0}^{(2)}+\frac{\sqrt{6}}{42}\left(A_{\tau 2}^{(2)}+A_{\tau-2}^{(2)}\right)\right] \cos ^{2} \alpha\right. \\
& \left.+\left[-\frac{4}{21} A_{\tau 0}^{(2)}+\frac{\sqrt{6}}{14}\left(A_{\tau 2}^{(2)}+A_{\tau-2}^{(2)}\right)\right] \sin ^{2} \alpha\right\} B_{x z}^{*} \alpha_{z x} .
\end{aligned}
$$

Then an angle $\alpha$ may exist given by

$$
\alpha=\tan ^{-1} \sqrt{\frac{A_{00}^{(2)}+\sqrt{6} A_{02}^{(2)}}{4 A_{00}^{(2)}-3 \sqrt{6} A_{02}^{(2)}}}
$$


at which the effective anisotropic susceptibility with $\tau=0$ vanishes. Therefore only the rotational decay with $\tau=2$ contributes to the effective anisotropic susceptibility. Since the isotropic part of the effective susceptibility is nonzero, the TRA CARS signal for mode 2 will contain the isotropic decay and the anisotropic decay with $\tau=2$ in this polarization configuration.

While the theoretical treatment in this section describes the case of rotational diffusion of the chromophore, the TRA CARS technique is able to probe other regimes of rotational dynamics as well. Recently, inertial components of rotational relaxations have been detected by ultrafast time-resolved fluorescence anisotropy measurements. ${ }^{22,23}$ Inertial motions (or other models of rotational dynamics) can be incorporated into the theory of TRA CARS by writing the third-order nonlinear susceptibility as

$$
\chi^{(3)}(t)=N(0) e^{-k_{f} t}\left[\chi_{I}^{(3)}+\chi_{A}^{(3)} C(t)\right],
$$

where $N(0)$ is the initially generated number density, $\chi_{I}^{(3)}$ and $\chi_{A}^{(3)}$ are the initial isotropic and anisotropic components of the third-order susceptibility (discussed in Sec. II E), and $C(t)$ is a rotational correlation function. Derivation of an expression for $C(t)$ would require incorporation of a model for the rotational dynamics into Eqs. (9) and (10).

\section{Application to time-resolved spontaneous Raman scattering}

Since the differential Raman scattering cross section is related directly to the imaginary part of the susceptibility Im $\chi_{i j k l}$, the formalism developed here can be applied directly to time-resolved Raman scattering. This theory was recently developed by one of us. ${ }^{12}$ Its application is discussed in this section.

Raman scattering is described by the third-order nonlinear susceptibility

$$
\chi_{i j k l}\left(-\omega_{0}, \omega_{s}, \omega_{0},-\omega_{s}\right)=C^{\prime \prime} \alpha_{i j}^{*} \alpha_{k l},
$$

where $\omega_{0}$ and $\omega_{s}$ are the frequencies of the input light and the scattered light, respectively, and $C^{\prime \prime}$ is a constant. The Raman polarizability tensor $\alpha_{i j}$ is defined in Eq. (6). Caution must be exercised, however, with regard to the indices $i j k l$. In the definition of $\alpha_{i j}$ in Eq. (6), the indices $i$ and $k$ denote the transition induced by the incident light $\left(\omega_{0}\right)$, while the indices $j$ and $l$ denote the transition generating scattered light $\left(\omega_{s}\right)$. (In some papers, $\alpha_{i j}$ is defined such that $i$ denotes the scattered light and $j$ denotes the incident light.) The formalism developed here can then be applied directly to time-resolved Raman scattering ${ }^{12}$ by replacing the anti-Stokes scattering tensor $\beta_{i j}^{*}$ with $\alpha_{i j}^{*}$.

In particular, we consider a time-resolved Raman experiment where the polarization of the pump pulse $E_{p}\left(\omega_{\mathrm{ex}}\right)$ forms an angle $\theta$ with respect to the polarization of the Raman excitation pulse $E\left(\omega_{0}\right)$ as shown in Fig. 1. In this case, the effective susceptibility is given by

$$
\begin{aligned}
& \chi_{\mathrm{eff}}^{(\mathrm{H})}=\chi_{1111} \cos ^{2} \theta+\chi_{2222} \sin ^{2} \theta, \\
& \chi_{\mathrm{eff}}^{(1)}=\chi_{1212} \cos ^{2} \theta+\chi_{2323} \sin ^{2} \theta
\end{aligned}
$$

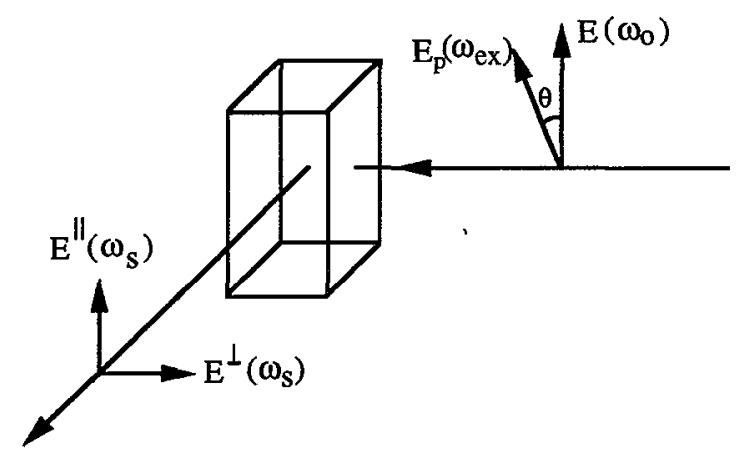

FIG. 1. Polarization configuration for time-resolved Raman scattering. $E_{p}\left(\omega_{e x}\right)$ is the polarization of the pump pulse. Raman scattering is generated by the vertically polarized probe pulse $E\left(\omega_{0}\right)$. The Stokes-shifted scattered light at $90^{\circ}$ has polarization parallel $E^{\text {ll }}\left(\omega_{s}\right)$, or perpendicular $E^{1}\left(\omega_{s}\right)$, to $E\left(\omega_{0}\right)$.

for scattered light with polarization parallel and perpendicular to $E\left(\omega_{0}\right)$ (in a $90^{\circ}$ scattering geometry). The Raman signal will in general be dependent on orientation, and reorientation may contribute to the time dependence in time-resolved Raman experiments. In some circumstances, these rotational effects can be eliminated, however, at an appropriately chosen angle $\theta$. For example, since $\chi_{2222,2 \tau}=$ $-\frac{1}{2} \chi_{1111,2 \tau}$ (see Table II), the parallel anisotropic component of the Raman scattering vanishes at the magic angle $\theta_{m}=54.7^{\circ}$. However, in general, the perpendicular anisotropic component of the scattering does not vanish at the magic angle. For example, for modes 1 and 2 discussed in the preceding section, $\chi_{2323,2 \tau}=-2 \chi_{1212,2 \tau}$ (for spontaneous Raman scattering $G_{12}=G_{21}$ if the scattering tensor is real). Hence, the perpendicular anisotropic component for these modes vanishes at $90^{\circ}-\theta_{m}$.

As a general conclusion, it is important to note that rotational effects may contribute to the time dependence of time-resolved Raman signals. This time dependence might be attributed to vibrational evolution if anisotropic contributions are not considered.

\section{E. The initial anisotropic susceptibility}

In the above, we have discussed the time evolution of TRA CARS signals. In addition to time scans, the TRA CARS spectrum can also be measured at a fixed delay time. The TRA CARS spectrum is in general different from the normal CARS spectrum due to contributions of the anisotropic part of the third-order nonlinear susceptibility. Hence the TRA CARS spectrum can provide new spectroscopic information which is contained in the anisotropic susceptibility constant $G_{L L^{\prime}}(\tau)$.

In this section, we discuss the anisotropic susceptibility generated immediately after excitation by the pump pulse. Measurement at time delays immediately following the excitation pulse profile yields the initial anisotropic susceptibility. The expression for the initial susceptibility is determined by the initial distribution and is given by 


$$
\begin{aligned}
\chi_{i j k l}= & N(0)\left(\sum_{L=0}^{2} S_{i j k l, L} G_{L}\right. \\
& \left.+\sum_{L=0}^{2} \sum_{L^{\prime}=0}^{2} \frac{\sqrt{6}}{5} S_{i j k l, L L^{\prime}} C_{L L^{\prime}}^{0}\right),
\end{aligned}
$$

where $N(0)$ is the initial number density of the excited state or the ground hole, and the initial anisotropic constants $G_{L L}^{0}$, are defined by

$$
\begin{aligned}
G_{L L^{\prime}}^{0}= & \sum_{T=2}^{2} F_{T}^{(2)} \sum_{M=-L}^{L} \sum_{M^{\prime}=-L}^{4}(-1)^{M^{\prime}} \\
& \times C\left(L^{\prime} L 2 ;-M^{\prime} M T\right) \beta_{M}^{(L) *} \alpha_{M^{\prime}}^{\left(L^{\prime}\right)},
\end{aligned}
$$

where $F_{T}^{(2)}$ is given in Eq. (17). (Dephasing times are assumed here to be much faster than the time scale of rotational dynamics.) By comparison to the expression for $G_{L L^{\prime}}(\tau)$ in Eq. (36), it can be seen that the initial anisotropic constant $G_{L L^{\prime}}^{0}$ is obtained by replacing $A_{2 \tau, T}^{(2)}$ with $F_{T}^{(2)}$ in $G_{L L^{\prime}}(\tau)$. Hence, Table III gives the initial anisotropic constant $G_{L L^{\prime}}^{0}$ when $F_{T}^{(2)}$ replaces $A_{2 \tau T}^{(2)}$.

The third-order susceptibility at the initial time can be written in a general form

$\chi_{0}^{(3)}=\chi_{I}^{(3)}+\chi_{A}^{(3)}$,

where

$$
\begin{aligned}
\chi_{I}^{(3)}= & d \cdot N(0) \sum_{L=0}^{2}\left(\sum_{i j k l} e_{3 i}^{*} e_{0 j} e_{1 k} e_{2 i}^{*} S_{i j k l, L}\right) G_{L}, \\
\chi_{A}^{(3)}= & d \cdot N(0) \\
& \times \sum_{L=0}^{2} \sum_{L^{\prime}=0}^{2}\left(\sum_{i j k l} e_{3 i}^{*} e_{0 j} e_{1 k} e_{2 l}^{*} \frac{\sqrt{6}}{5} S_{i j k l, L L^{\prime}}\right) G_{L L^{\prime}}^{0}
\end{aligned}
$$

Here $N(0)$ is the initial number density of the excited state or the ground state hole; $d$ is a constant depending on the frequencies [see Eq. (4)]; and $e_{3}, e_{0}, e_{1}$, and $e_{2}$ are the unit vectors for the fields $E\left(\omega_{3}\right), E\left(\omega_{0}\right), E\left(\omega_{1}\right)$, and $E\left(\omega_{2}\right)$. Thus, the initial third-order susceptibility can be written

$$
\begin{aligned}
\chi_{0}^{(3)}= & a_{0} G_{0}+a_{1} G_{1}+a_{2} G_{2}+b_{02} G_{02}^{0}+b_{20} G_{20}^{0}+b_{11} G_{11}^{0} \\
& +b_{12} G_{12}^{0}+b_{21} G_{21}^{0}+b_{22} G_{22}^{0},
\end{aligned}
$$

where

$$
\begin{aligned}
& a_{L}=d \cdot N(0) \sum_{i j k l} e_{3 i}^{*} e_{0 j} e_{1 k} e_{2 l}^{*} S_{i j k l, L}, \\
& b_{L L^{\prime}}=d \cdot N(0) \sum_{i j k l} e_{3 i}^{*} e_{0 j} e_{1 k} e_{2 l}^{*} \frac{\sqrt{6}}{5} S_{i j k l, L L^{\prime}}
\end{aligned}
$$

The nine molecular parameters $G_{L}$ and $G_{. L L^{\prime}}^{0}$ are independent of experimental polarization, while $a_{L}$ and $b_{L L^{\prime}}$ are polarization parameters selected in the experimental setup. The set of parameters $\left(G_{L}, G_{L L^{\prime}}^{0}\right)$ can be regarded as a nine-dimensional vector $\mathbf{G}$. For any measurement, $\chi^{(3)}$ is given by the dot product of $\mathbf{G}$ with the vector $\left(a_{L}, b_{L L^{\prime}}\right)$ in
TABLE IV. Polarization configurations in spontaneous Raman anisotropic susceptibility measurements. ${ }^{\mathrm{a}}$

\begin{tabular}{cccc}
\hline \hline Case & $E_{p}\left(\omega_{\mathrm{ex}}\right)$ & $E\left(\omega_{0}\right)$ & $E\left(\omega_{s}\right)$ \\
\hline$(1)$ & $\uparrow$ & $\uparrow$ & $\uparrow$ \\
$(2)$ & $\rightarrow$ & $\uparrow$ & $\uparrow$ \\
$(3)$ & $\uparrow$ & $\uparrow$ & $\vec{\uparrow}$ \\
$(4)$ & $\uparrow$ & $\rightarrow$ & $\odot$ \\
$(5)$ & $\uparrow$ & $\overrightarrow{0}$ & $\uparrow$ \\
$(6)$ & $\uparrow$ & $\oplus$ & $\odot$ \\
$(7)$ & $\uparrow$ & $\oplus$ & $\odot$ \\
\hline \hline
\end{tabular}

${ }^{\mathrm{a}} E_{p}\left(\omega_{\mathrm{ex}}\right)$ is the polarization of the pump pulse, $E\left(\omega_{0}\right)$ is the Raman excitation polarization, and $E\left(\omega_{s}\right)$ is the polarization of scattered light.

the same space. By making nine measurements of $\chi^{(3)}$ with linearly independent values of $a_{L}$ and $b_{L L^{\prime}}$, one could determine each of the components $G_{L}$ and $G_{L L}^{0}$. Eight of the possible polarization configurations with collinear pump and probe propagation are shown in Table II.

It is noteworthy that the anisotropic third-order susceptibility consists of nine independent parameters instead of the three in an isotropic system. This means that the anisotropic CARS method can in principle yield more information regarding, e.g., the symmetry of vibrations and excited states, than can be obtained by isotropic CARS. Similarly, anisotropic time-resolved Raman scattering can generate new information. For example, the Raman scattering tensor might be determined completely by this technique in a randomly oriented molecular system. Since anisotropic CARS or Raman experiments can probe both ground and excited state anisotropies, the initial susceptibility in either ground or excited states can be measured, depending on experimental conditions.

To measure the nine parameters $G_{L}$ and $G_{L L^{\prime}}^{0}$, we need to specify the polarizations of the Raman experiment. Among the possible cases for a $90^{\circ}$ scattering configuration with $E\left(\omega_{0}\right)$ propagating parallel to $E_{p}\left(\omega_{\mathrm{ex}}\right)$ are the following:

(1) $E_{p}\left(\omega_{\mathrm{ex}}\right), E\left(\omega_{0}\right)$, and $E\left(\omega_{s}\right)$ polarized linearly with parallel polarization;

(2) $E_{p}\left(\omega_{\mathrm{ex}}\right), E\left(\omega_{0}\right)$, and $E\left(\omega_{s}\right)$ polarized linearly and $E\left(\omega_{0}\right)$ and $E\left(\omega_{s}\right)$ parallel; $E_{p}\left(\omega_{\mathrm{ex}}\right)$ perpendicular;

(3) $E_{p}\left(\omega_{\mathrm{ex}}\right), E\left(\omega_{0}\right)$, and $E\left(\omega_{s}\right)$ polarized linearly and $E_{p}\left(\omega_{\mathrm{ex}}\right)$ and $E\left(\omega_{0}\right)$ parallel; $E\left(\omega_{s}\right)$ perpendicular;

(4) $E_{p}\left(\omega_{\mathrm{ex}}\right), E\left(\omega_{0}\right)$, and $E\left(\omega_{s}\right)$ polarized linearly and $E_{p}\left(\omega_{\mathrm{ex}}\right)$ and $E\left(\omega_{s}\right)$ parallel; $E\left(\omega_{0}\right)$ perpendicular;

(5) $E_{p}\left(\omega_{\mathrm{ex}}\right), E\left(\omega_{0}\right)$, and $E\left(\omega_{s}\right)$ polarized linearly, but mutually perpendicular to each other;

(6) $E_{p}\left(\omega_{\mathrm{ex}}\right)$ and $E\left(\omega_{s}\right)$ polarized linearly and parallel; $E\left(\omega_{0}\right)$ circularly polarized;

(7) $E_{p}\left(\omega_{\mathrm{ex}}\right)$ linearly polarized, $E\left(\omega_{0}\right)$ circularly polarized, and $E\left(\omega_{s}\right)$ linearly polarized, but perpendicular to $E_{p}\left(\omega_{\mathrm{ex}}\right)$ and $E\left(\omega_{0}\right)$.

These cases are illustrated schematically in Table IV. In the cases of linearly polarized $E\left(\omega_{0}\right)$ [cases (1)-(5)], the values of $a_{L}$ and $b_{L L^{\prime}}$ can be obtained directly from the 


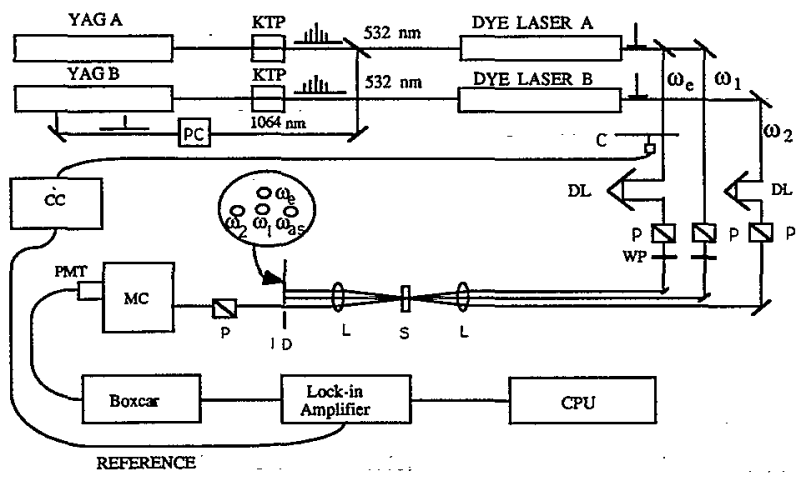

FIG. 2. The experimental setup for TRA CARS measurements. PCPockels cell; C-chopper; CC-chopper controller; DL-delay line; P-polarizer; WP-half-wave plate; L-lens; S-sample; ID-iris diaphragm; MC-monochromator; PMT-photomultiplier tube; CPUcomputer.

values of $S_{i j k l, L}$ and $S_{i j k l, L L^{\prime}}$ in Table II since $a_{L}=S_{i j k l, L}$ and $b_{L L^{\prime}}=S_{i j k l, L L^{\prime}}$ for these cases. For the cases with circularly polarized $E\left(\omega_{0}\right)$, one can write

$$
\begin{aligned}
& \chi_{\mathrm{cir}}^{\|}=\frac{1}{2}\left(\chi_{1111}+\chi_{2121}\right), \\
& \chi_{\mathrm{cir}}^{1}=\frac{1}{2}\left(\chi_{1212}+\chi_{2323}\right)
\end{aligned}
$$

for cases (6) and (7), respectively. Thus $a_{L}$ and $b_{L L^{\prime}}$ can be obtained easily from Table II for cases (6) and (7). For Raman scattering, if the scattering tensor is real, $G_{02}^{0}=G_{20}^{0}$ and $G_{12}^{0}=G_{21}^{0}$, and the number of independent parameters reduces to seven. The seven polarization configurations in cases (1) and (7) discussed above represent five independent measurements. Additional independent measurements can be made in other scattering geometries.

\section{TIME-RESOLVED ANISOTROPIC CARS}

In this section, we describe the experimental implementation of TRA CARS. Three types of measurements are described depending on the experimental polarization configuration. In each case, TRA CARS decays for sulforhodamine 640 are presented to illustrate the time dependence of the CARS signal.

\section{A. Experimental method}

The experimental setup is shown in Fig. 2. The laser system, which has been described elsewhere, ${ }^{24}$ employs two $Q$-switched, mode-locked Nd:YAG lasers to pump two cavity-dumped dye lasers. When the same $38 \mathrm{MHz}$ radiofrequency oscillator is used to drive both mode lockers, the timing jitter between the two Nd:YAG lasers is $\sim 50 \mathrm{ps.}^{24}$ This jitter is transmitted to the synchronously pumped dye lasers. In order to reduce the jitter between pump and probe, pulses from YAG $A$ were regeneratively amplified in YAG $B$ to ensure pulse synchronization. This method reduces the pump-probe jitter to $<10$ ps. Pulses are obtained at a repetition rate of $\sim 1000 \mathrm{~Hz}$. The time resolution of the system is $\sim 50 \mathrm{ps}$. The excitation laser beam was chopped at precisely half the laser repetition rate to alternately block and transmit the pump pulses. CARS signals
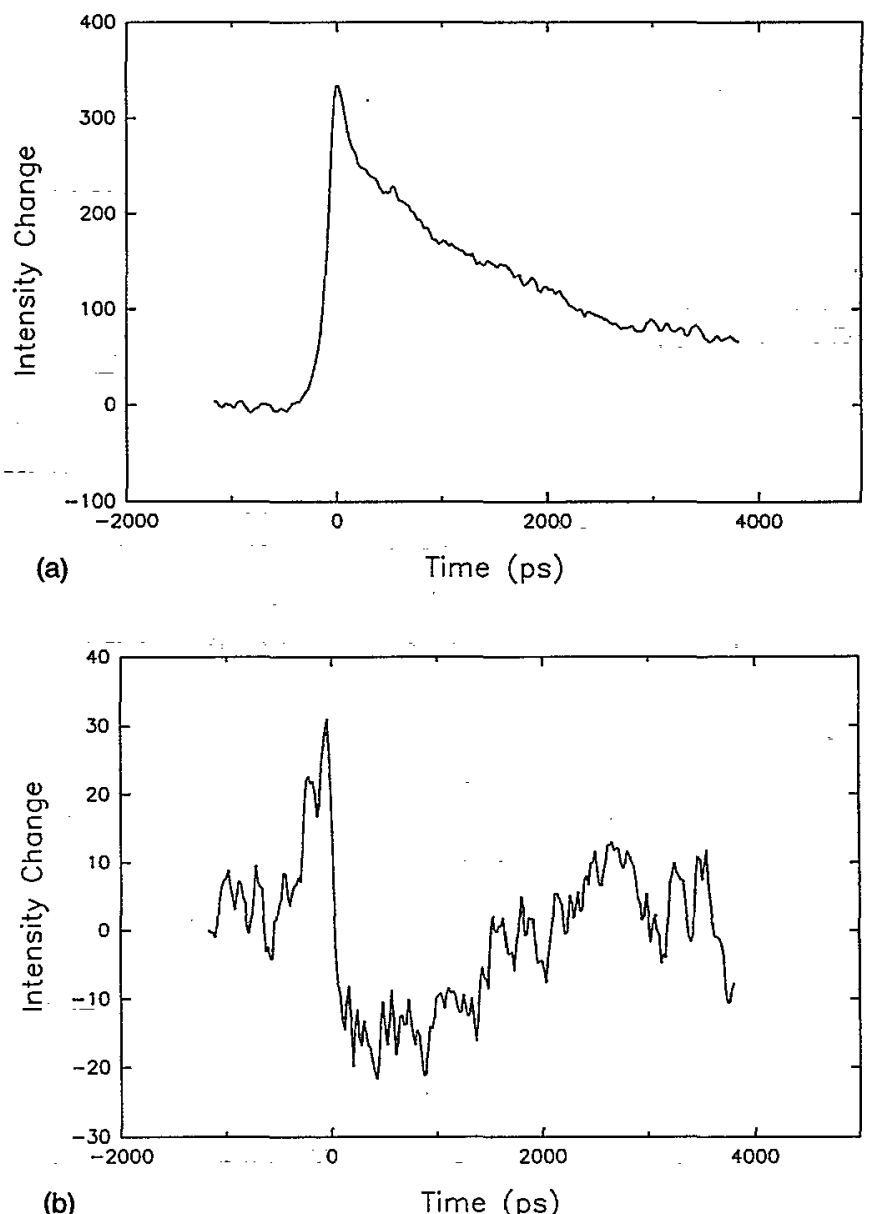

FIG. 3. The change in TRA CARS signals for components (a) $\chi_{2112}$ and (b) $\chi_{1221}$ for sulforhodamine 640 in ethylene glycol. $\lambda_{1}=\lambda_{e}=580 \mathrm{~nm}$; $\lambda_{2}=641.2 \mathrm{~nm}$; Raman shift $1646 \mathrm{~cm}^{-1}$.

were processed with a gated integrator and lock-in amplifier to generate the difference signal induced by the excitation pulse. Trigger pulses for the $Q$-switch modulators, the cavity dumpers, and the gated integrator were generated by a pulse generator built in house. ${ }^{24}$ In these experiments, two laser beams $\left(\omega_{1}\right.$ and $\left.\omega_{2}\right)$ were used to generate CARS signals with frequency degeneracy $\omega_{0}=\omega_{1}$. Sulforhodamine 640 (sulforhodamine 101) was obtained from Exciton.

\section{B. Results and discussion}

Three different polarization configurations were used to generate and detect anisotropic CARS signals. In the first configuration, a single third-order nonlinear susceptibility component is measured. Table II shows the polarization configurations for measuring the components $\chi_{2112}$, $\chi_{1221}, \chi_{2222}$, and $\chi_{1111}$. The signal detected in this experiment is the change induced by the pump pulse

$$
\left|\chi_{0}+\Delta \chi(t)\right|^{2}-\left|\chi_{0}\right| \approx 2 \operatorname{Re}\left[\chi_{0}^{*} \Delta \chi(t)\right],
$$

where $\Delta \chi$ is small relative to $\chi_{0}$, the susceptibility before excitation. The difference signal contains both the isotropic and anisotropic decays. This is an optical heterodyne mea- 

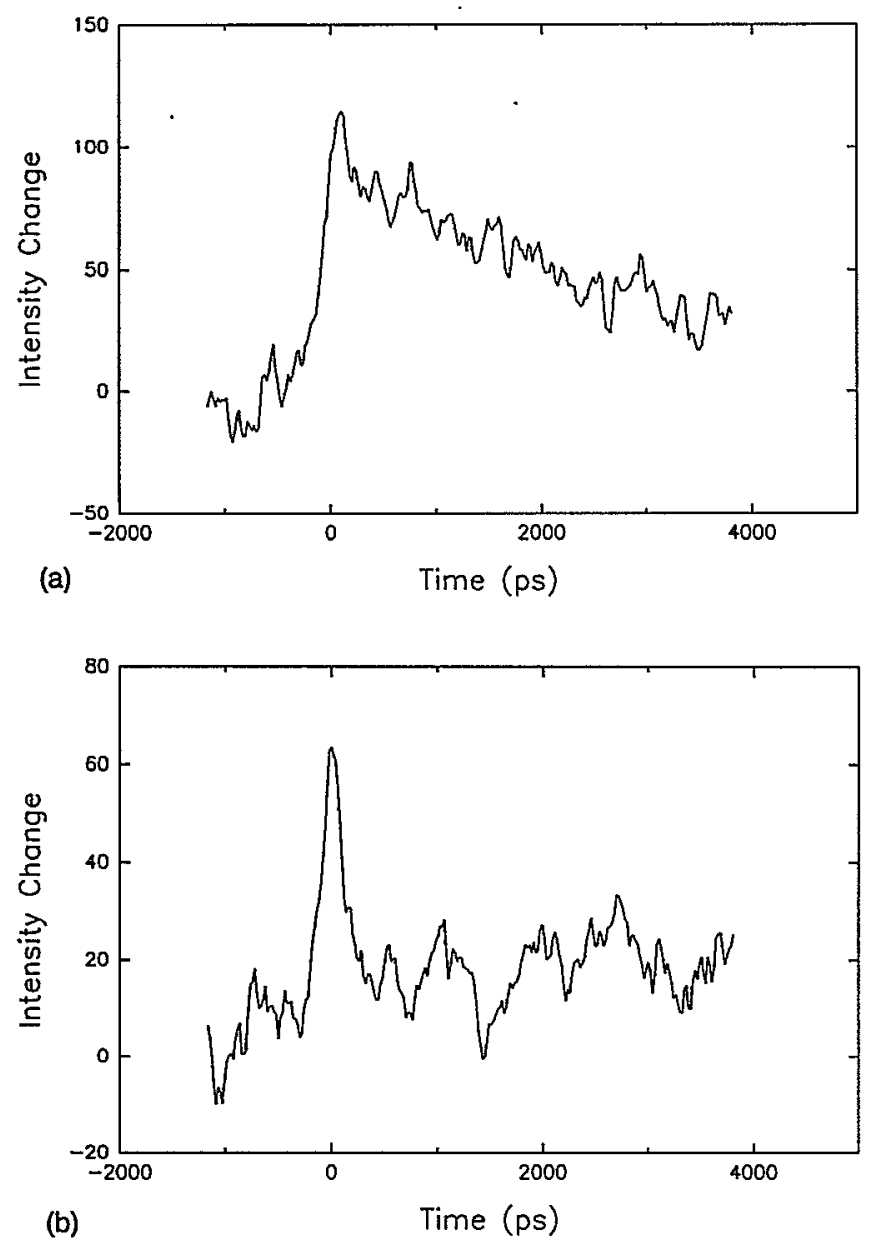

FIG. 4. The change in TRA CARS signals for components (a) $\chi_{2222}$ and (b) $\chi_{1111}$. Other experimental parameters are the same as in Fig. 3.

surement with a local oscillator field generated by $\chi_{0}$. Since these signals are linear in the susceptibility change $\Delta \chi$, such measurements could be used to determine anisotropies as in Eq. (37). At present, however, these signals are too noisy for anisotropy measurements.

Decays for the third-order susceptibility components $\chi_{1221}$ and $\chi_{2112}$ are shown in Fig. 3 for sulforhodamine 640 in ethylene glycol $\left(\lambda_{1}=580 \mathrm{~nm}, \lambda_{2}=641.3 \mathrm{~nm}\right.$, and Raman shift $\left.=1647 \mathrm{~cm}^{-1}\right)$. The time-dependent response is strikingly different. Since the isotropic components are equal $\chi_{1221,00}=\chi_{2112,00}$, the marked difference between the $\chi_{1221}$ and $\chi_{2112}$ decays shows that the anisotropic component of the third-order susceptibility contributes strongly to these signals. In particular, the increase in $\chi_{2112}$ is much
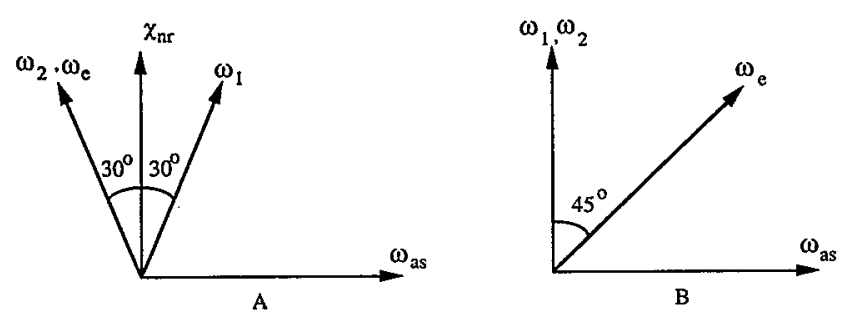

FIG. 5. TRA CARS polarization configurations. (A) Nonresonant background free TRA CARS. (B) TRA CARS polarization spectroscopy.

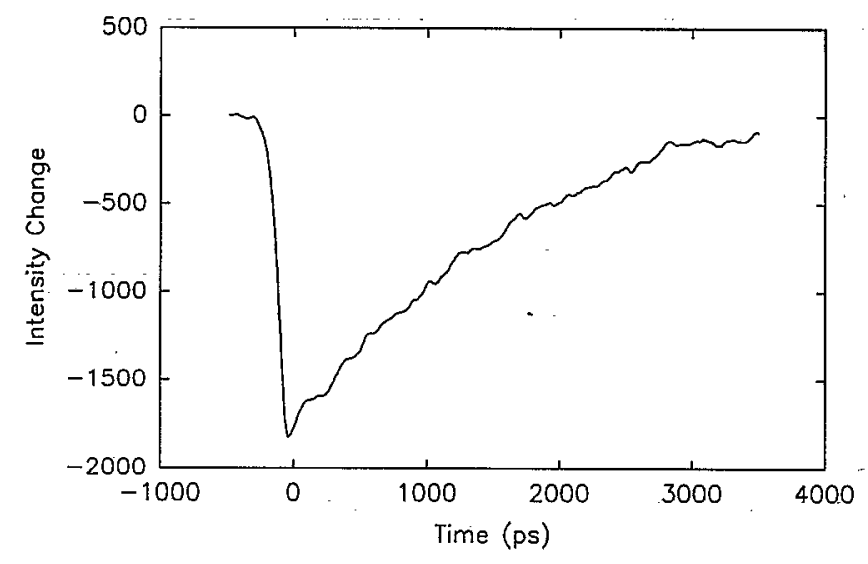

FIG. 6. Nonresonant background-free TRA CARS of sulforhodamine 640 in ethylene glycol at Raman shift $164.6 \mathrm{~cm}^{-1}$. The recovery time constant is $1450 \mathrm{ps}$.

stronger than the change in $\chi_{1221}$. The sharp increase at $t \approx 0$ in $\chi_{1221}$, which may result from coherence between $E\left(\omega_{\mathrm{ex}}\right)$ and $E\left(\omega_{1}\right)$, is followed by a weak decrease in signal. Similarly, Fig. 4 shows the change in the TRA CARS signals for $\chi_{2222}$ and $\chi_{1111}$. While $\chi_{1111}$ shows no detectable change after a spike at $t \approx 0, \chi_{2222}$ increases markedly and decays. A detailed analysis of the signal changes is not possible without a more complete knowledge of the contributions (real and imaginary) to the CARS susceptibilities and will not be attempted in this paper. Rather, the motivation here is to demonstrate the importance of anisotropic contributions to the CARS signals.

The second type of TRA CARS configuration is one that suppresses the nonresonant susceptibility $\chi_{\mathrm{NR}}^{(3)}$. For an isotropic system, it is well known that the nonresonant background is zero when the effective susceptibility is ${ }^{25}$

$$
\chi_{\text {eff }}=\chi_{1111}-3 \chi_{2112} \text {. }
$$

A background-free configuration with $E\left(\omega_{1}\right)$ oriented $+30^{\circ}$ and $E\left(\omega_{2}\right)$ oriented $-30^{\circ}$ from the $Z$ axis and $E\left(\omega_{a s}\right)$ along the $X$ axis is shown in Fig. 5(A). In TRA CARS, the induced anisotropic susceptibility is given by Eq. (59) when $E_{p}\left(\omega_{e x}\right)$ is parallel to $E_{p}\left(\omega_{1}\right)$. When

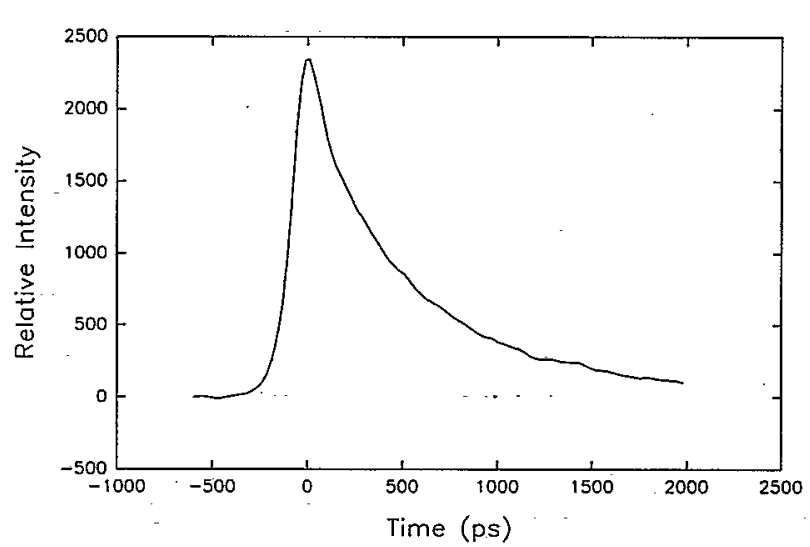

FIG. 7. TRA CARS polarization spectroscopy decay of sulforhodamine 640 in ethylene glycol at Raman shift $1646 \mathrm{~cm}^{-1} ; \lambda_{\mathrm{ex}}=\lambda_{1}=580 \mathrm{~nm}$; $\lambda_{2}=641.2 \mathrm{~nm}$. 


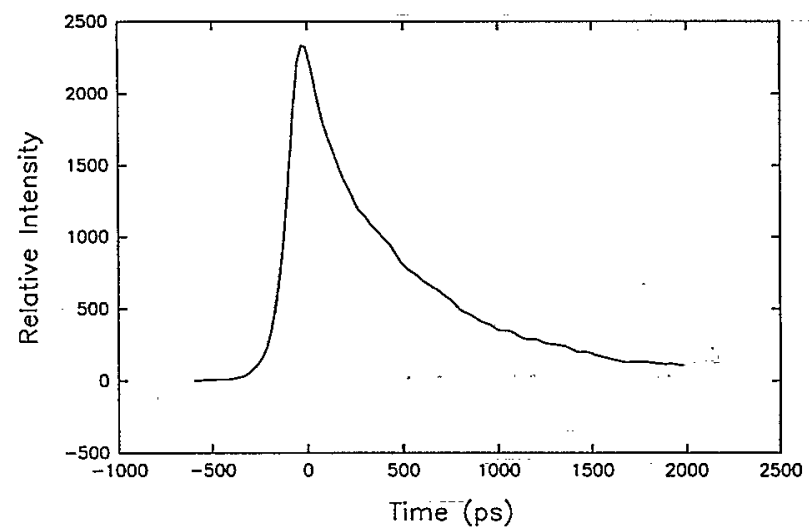

FIG. 8. TRA CARS polarization spectroscopy decay of sulforhodamine 640 in ethylene glycol at Raman shift $1498 \mathrm{~cm}^{-1} ; \lambda_{\mathrm{ex}}=\lambda=580 \mathrm{~nm}$; $\lambda_{2}=635.2 \mathrm{~nm}$.

$E_{p}\left(\omega_{\mathrm{ex}}\right)$ is not parallel to $E\left(\omega_{1}\right)$, the anisotropic part of the third-order susceptibility may contain terms representing eight polarization components (see Table II). However, since the isotropic part can still be described by Eq. (59), the nonresonant background generated by the solvent will be suppressed. Figure 6 shows a nonresonant background-free TRA CARS scan, where $E_{p}\left(\omega_{\mathrm{ex}}\right)$ is parallel to $E\left(\omega_{1}\right)$ for sulforhodamine 640 in ethylene glycol at a Raman shift of $1646 \mathrm{~cm}^{-1}$. The absolute value of the effective susceptibility decreases upon excitation and recovers with a time constant of 1450 ps. This signal includes both isotropic and anisotropic components.

A third TRA CARS configuration is shown in Fig. 5(B), where $E\left(\omega_{1}\right)$ and $E\left(\omega_{2}\right)$ are parallel, $E\left(\omega_{a s}\right)$ is perpendicular to $E\left(\omega_{1}\right)$, and $E_{p}\left(\omega_{\mathrm{ex}}\right)$ is $45^{\circ}$ from $E\left(\omega_{1}\right)$. This configuration is analogous to that in polarization (or anisotropic absorption) spectroscopy. ${ }^{26,27}$ In the absence of the excitation field $E_{p}\left(\omega_{\mathrm{ex}}\right)$, the signal field $E\left(\omega_{a s}\right)$ is zero since the CARS field is perpendicular to $E\left(\omega_{a s}\right)$. However, $E\left(\omega_{a s}\right)$ is nonzero after the sample system is excited by the excitation field $E_{p}\left(\omega_{\mathrm{ex}}\right)$. The effective susceptibility in this case is

$$
\begin{aligned}
\chi_{\mathrm{eff}}= & \chi_{1111}-\chi_{2222}+\chi_{1221}-\chi_{2112}+\chi_{1212} \\
& -\chi_{2121}+\chi_{1122}-\chi_{2211}
\end{aligned}
$$

From Table II, we can see that the isotropic part of the TRA CARS susceptibility is zero and the effective susceptibility contains only the anisotropic part. For a two-level system where excited and ground states have the same rotational constants, the effective susceptibility can be written

$$
\begin{aligned}
\chi_{\mathrm{eff}}^{(i)}= & \sum_{\tau=-2}^{2} \exp \left[-\left(k_{f}+E_{2 \tau}\right) t\right] a_{2 \tau}^{(i)}(0)\left(-\frac{\sqrt{2}}{5}\right) \\
& \times\left[G_{20}^{(i)}(\tau)+G_{12}^{(i)}(\tau)+\frac{1}{\sqrt{7}} G_{22}^{(i)}(\tau)\right],
\end{aligned}
$$

where $k_{f}$ is the kinetic decay rate, $E_{2 \tau}$ is the rotational eigenvalues, $G_{L L^{\prime}}^{(i)}(\tau)$ 's are the anisotropic susceptibility constants for state $i$, and $a_{2 \tau}^{(i)}(0)$ 's are given in Eqs. (16)

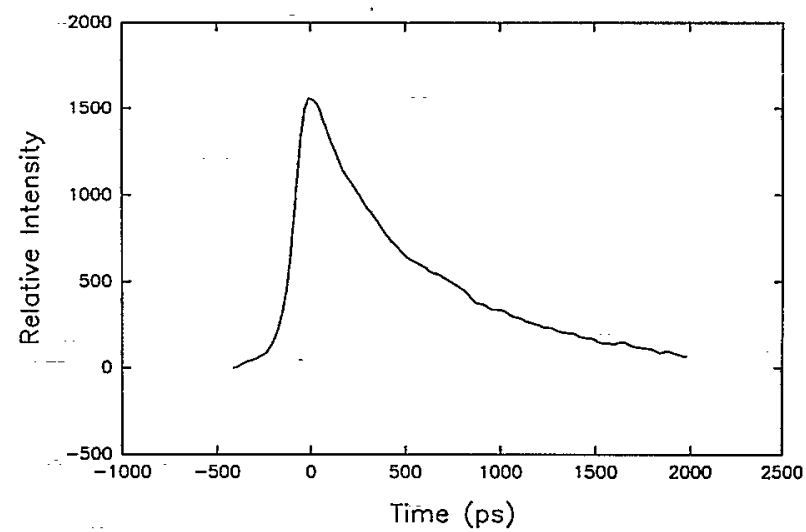

FIG. 9. TRA CARS polarization spectroscopy decay of sulforhodamine 640 in ethylene glycol at Raman shift $1359 \mathrm{~cm}^{-1} ; \lambda_{\mathrm{ex}}=\lambda_{1}=580 \mathrm{~nm}$; $\lambda_{2}=629.6 \mathrm{~nm}$.

and (17). The effective susceptibility contains five exponentials in general. If the excitation dipole is coincident with one of the principal rotation axes, the effective susceptibility reduces to two exponentials with $\tau=0$ and 2 [see Eq. (17) and tabulated values of $\left.A_{2 \tau T}\right]$.

Figures 7-10 show decays detected in the TRA CARS polarization configuration for sulforhodamine 640 vibrations at 1359,1498 , and $1646 \mathrm{~cm}^{-1}$ compared to a decay collected by conventional polarization spectroscopy. The decays are fitted to a single exponential function. The resulting decay times are listed in Table $\mathrm{V}$ for two solvents. In both solvents 1-butanol and ethylene glycol, the decay times for the 1498 and $1646 \mathrm{~cm}^{-1}$ vibrations are the same as those measured by polarization spectroscopy within experimental error. However, the decay time for the 1359 $\mathrm{cm}^{-1}$ vibration is clearly longer. As discussed above, the anisotropy decay may not be single exponential, but rather the mixture of two or more exponentials, and the anisotropic susceptibility constant $G_{L L^{\prime}}(\tau)$ may be vibration dependent in that the relative contribution of each rotational decay for $\tau=0, \pm 1, \pm 2$ to the TRA CARS decay depends on the vibration. Therefore the TRA CARS measurement may reveal that the anisotropic decays of SRG

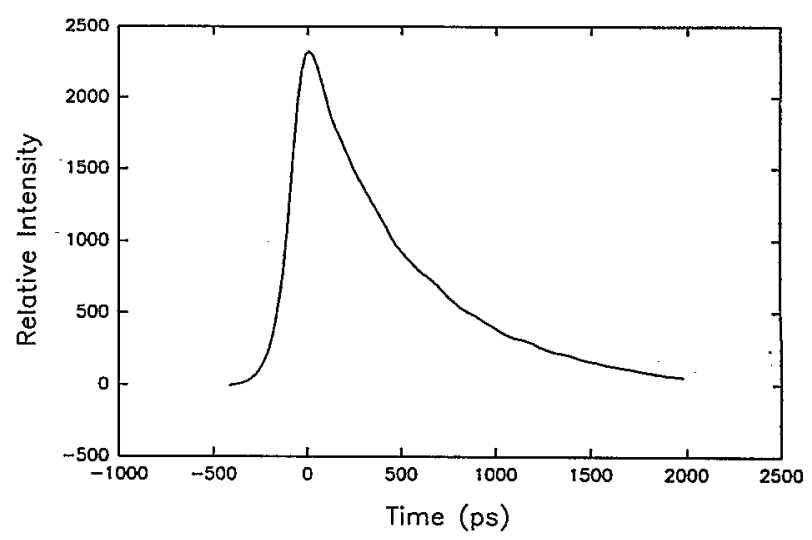

FIG. 10. Polarization spectroscopy decay of sulforhodamine 640 in ethylene glycol excited at $580 \mathrm{~nm}$ and probed at $629.6 \mathrm{~nm}$. 
TABLE V. Decay time constants determined by TRA CARS polarization spectroscopy and conventional polarization spectroscopy for sulforhodamine 640 .

\begin{tabular}{lllll}
\hline \hline & \multicolumn{3}{c}{ Vibrational mode } & \\
\cline { 2 - 4 } & $1359 \mathrm{~cm}^{-1}$ & $1498 \mathrm{~cm}^{-1}$ & $1646 \mathrm{~cm}^{-1}$ & Polarization spectroscopy \\
\hline SR640/1-butanol & $350 \pm 6 \mathrm{ps}$ & $296 \pm 8 \mathrm{ps}$ & $325 \pm 11 \mathrm{ps}$ & $311 \pm 7 \mathrm{ps}$ \\
SR640/ethylene glycol & $719 \pm 29 \mathrm{ps}$ & $606 \pm 41 \mathrm{ps}$ & $626 \pm 46 \mathrm{ps}$ & $594 \pm 16 \mathrm{ps}$ \\
\hline \hline
\end{tabular}

640 molecules are in fact multiple exponentials instead of single exponentials as previously assumed. ${ }^{28}$

The first type of TRA CARS experiment, which measures selected susceptibility tensor components, can in principle provide the most information about rotational dynamics, but suffers from the drawback that the TRA CARS signal measured by this method is noisy since the induced signal rides a much stronger background. The second type of experiment eliminates the nonresonant background. However, the isotropic decay may still be strong and the anisotropic component is not readily measured. The third type of experiment TRA CARS polarization spectroscopy measures the pure anisotropic decay and yields the best signal quality, even though the signal measured in this way is much weaker than in the other two methods. However, some information is lost because the effective susceptibility only contains three of the six anisotropic CARS constants as shown in Eq. (61).

\section{CONCLUSION}

In this paper, we have developed the formalism for the time-dependent anisotropic third-order susceptibility to describe TRA CARS decays. A comparison of the molecular information provided by TRA CARS and TRA Raman experiments may be useful. Since both techniques probe the anisotropic components of the third-order nonlinear optical susceptibility, the same information is in principle available from either method. However, one method or the other may be advantageous under certain circumstances. For example, since CARS signals are strong and directional, TRA CARS can be used to measure rotational dynamics in strongly fluorescent samples, such as dye molecules. On the other hand, TRA CARS experiments are restricted in practice to configurations with collinear pump and probe beams, which permit no more than eight independent measurements (see Table II). Since spontaneous Raman scattering can be collected at any scattering angle, the complete set of nine independent molecular parameters can be measured by TRA Raman scattering by a combination, e.g., of back scattering and $90^{\circ}$ scattering measurements. Thus, while TRA CARS may be more amenable to measurements of the anisotropic susceptibility, TRA Raman can generate a full set of molecular parameters.

We have discussed three types of TRA CARS experiments and illustrated each type with experimental results. The method discussed here can also be extended to other Raman spectroscopies such as CSRS and resonance Raman. Correspondingly, anisotropic components and rotational dynamics may contribute to the time dependence in time-resolved spontaneous or coherent Raman experiments that are intended to measure purely vibrational dynamics. These cannot always be eliminated by a "magic-angle" measurement. In future work, we plan to investigate further the possibility of separation of different rotational decays by TRA CARS as well as by TRA Raman and TRA CSRS spectroscopies.

\section{ACKNOWLEDGMENT}

This work was supported by a grant from the NIH.

${ }^{1}$ T. J. Chuang and K. B. Eisenthal, J. Chem. Phys. 57, 5094 (1972).

${ }^{2}$ M. Ehrenberg and R. Rigler, Chem. Phys. Lett. 14, 539 (1972).

${ }^{3}$ A. J. Cross, D. H. Waldeck, and G. R. Fleming, J. Chem. Phys. 78, 6455 (1983).

${ }^{4}$ A. Szabo, J. Chem. Phys. 81, 150 (1984).

${ }^{5}$ A. B. Myers and R. M. Hochstrasser, IEEE J. Quantum Electron. 22, 1482 (1986).

${ }^{6}$ C. Z. Wan, J. Qian, and C. K. Johnson, J. Phys. Chem. 94, 8417 (1990).

${ }^{7}$ C. Z. Wan, J. Qian, and C. K. Johnson, Biochemistry 30, 394 (1991).

${ }^{8}$ L. D. Favro, Phys. Res. 119, 53 (1960).

${ }^{9}$ G. R. Fleming, Chemical Applications of Ultrafast Spectroscopy (Oxford University, New York, 1986).

${ }^{10}$ B. J. Berne and R. Pecora, Dynamic Light Scattering (Wiley, New York, 1976).

${ }^{11}$ J. H. R. Clarke, in Advances in Infrared and Raman Spectroscopy, edited by R. J. H. Clark and R. E. Hester (Heyden, London, 1978), Vol. 4, pp. 109-193.

${ }^{12}$ C. Z. Wan, Chem. Phys. Lett. 203, 119 (1993),

${ }^{13} \mathrm{~J}$. W. Nibler and G. A. Pubanz, in Advances in Nonlinear Spectroscopy, Advances in Spectroscoy, edited by R. J. H. Clark and R. E. Hester (Wiley, New York, 1988), Vol. 15, pp. 1-50.

${ }^{14}$ M. D. Levenson, Introduction to Nonlinear Laser Spectroscopy (Academic, New York, 1982).

${ }^{15}$ W. T. Huntress, J. Chem. Phys. 48, 3524 (1968).

${ }^{16}$ T. Tao, Biopolymers 8, 609 (1969).

${ }^{17}$ R. N. Zare, Angular Momentum (Wiley, New York, 1988), p. 105.

${ }^{18} \mathrm{O}$. S. Mortensen and S. Hassig, in Advances in Infrared and Raman Spectroscopy, edited by R. J. H. Clark and R. E. Hester (Heyden, London, 1988), Vol. 6, pp. 1-60.

${ }^{19}$ B. Dick, Chem. Phys. 113, 131 (1987).

${ }^{20}$ R. N. Zare, Angular Momentum (Wiley, New York, 1988), p. 179.

${ }^{21}$ R. N. Zare, Angular Momentum (Wiley, New York, 1988), pp. 101103.

${ }^{22}$ A. B. Myers, M. A. Pereira, P. L. Holt, and R. M. Hochstrasser, J. Chem. Phys. 86, 5146 (1987).

${ }^{23}$ M. A. Pereira, P. E. Share, M. J. Sarisky, and R. M. Hochstrasser, J. Chem. Phys. 94, 2513 (1991).

${ }^{24}$ C. K. Johnson, J. M. Bostick, S. A. Mounter, K. L. Ratzlaff, and D. E. Schloemer, Rev. Sci. Instrum. 59, 2375 (1988); J. M. Bostick, S. A. Mounter, and C. K. Johnson, Opt. Commun. 69, 54 (1988).

${ }^{25} \mathrm{R}$. Brakel and F. W. Schneider, Advances in Nonlinear Spectroscopy, Advances in Spectroscopy, edited by R. J. H. Clark and R. E. Hester (Wiley, New York, 1988), Vol. 15, pp. 149-192.

${ }^{26}$ C. V. Shank and E. P. Ippen, Appl. Phys. Lett. 26, 62 (1975).

${ }^{27}$ D. Waldeck, A. J. Cross, D. B. McDonald, and G. R. Fleming, J. Chem. Phys. 74, 3381 (1981).

${ }^{28}$ P. Myslinski and D. Wieczorek, J. Chem. Phys. 92, 969 (1990). 